\title{
Fungos micorrízicos arbusculares e vermicomposto na aclimatação de Alpinia purpurata (Viell.) Schum e Zingiber spectabile Griff. (Zingiberaceae)
}

\author{
Maryluce Albuquerque da Silva ${ }^{1}$, Fábio Sérgio Barbosa da Silva ${ }^{1}$, Adriana Mayumi Yano-Melo ${ }^{1}$, \\ Natoniel Franklin de Melo² e Leonor Costa Maia ${ }^{1,3}$
}

Recebido em 8/03/2005. Aceito em 17/08/2005

\begin{abstract}
RESUMO - (Fungos micorrízicos arbusculares e vermicomposto na aclimatação de Alpinia purpurata (Viell.) Schum e Zingiber spectabile Griff. (Zingiberaceae)). Visando selecionar fungos micorrízicos arbusculares (FMA) e substratos para produção de mudas micropropagadas de alpinia (Alpinia purpurata) e sorvetão (Zingiber spectabile), conhecidas espécies ornamentais tropicais, foram inoculadas mudas com 1.500 propágulos infectivos de Gigaspora albida, Glomus etunicatum e Acaulospora longula, em recipientes contendo: (a) solo; (b) solo e vermicomposto (3:1) e (c) solo, areia e vermicomposto (2:2:1). O delineamento foi inteiramente casualizado em fatorial de: quatro tratamentos de inoculação (três com FMA e controle não inoculado), três substratos, oito repetições, para cada vegetal. Após 70 (Z. spectabile) e 100 (A. purpurata) dias da inoculação, parâmetros de crescimento da planta foram avaliados tais como altura, número de folhas e perfilhos, massa seca da parte aérea e subterrânea, área foliar, e ainda a colonização micorrízica e a esporulação dos FMA. Não houve interações significativas entre os FMA e os substratos testados. Em A. purpurata os dois substratos com vermicomposto promoveram maior desenvolvimento; em Z. spectabile, apenas o substrato com maior proporção do fertilizante orgânico induziu crescimento diferenciado. A aplicação de adubo reduziu a colonização micorrízica nos dois hospedeiros e a esporulação de A. longula. Na fase de aclimatação, o uso de substratos orgânicos favorece a formação de mudas vigorosas de alpinia e sorvetão, mas pode ser prejudicial para a comunidade de FMA.
\end{abstract}

Palavras-chave: micropropagação, Glomeromycota, flores tropicais

ABSTRACT - (Arbuscular mycorrhizal fungi and the use of vermicompost on the acclimatization of Alpinia purpurata (Viell.) Schum and Zingiber spectabile Griff. (Zingiberaceae)). To select AMF and substrates that enhance production of micropropagated seedlings of Alpinia purpurata and Zingiber spectabile, tropical ornamental species, seedlings were inoculated with 1,500 infective propagules of Gigaspora albida, Glomus etunicatum and Acaulospora longula, in pots containing: (a) soil; (b) soil and vermicompost (3:1); (c) soil, sand and vermicompost (2:2:1). The experimental design was completely randomized in a factorial of: four inoculation treatments (three with AMF and uninoculated control), three substrates, with eight replicates for each plant species. After 70 (Z. spectabile) and 100 (A. purpurata) days of inoculation, plant growth parameters such as height, leaf and offshoots number, dry mass of shoots and roots, leaf area, as well as mycorrhizal colonization, AMF sporulation were evaluated. Significant interactions were not observed between AMF and substrates. In A. purpurata, the two substrates with vermicompost promoted growth, while in Z. spectabile only the substrate with higher proportion of this compound enhanced plant growth. Use of vermicompost reduced mycorrhizal colonization in both hosts and the sporulation of A. longula. During the acclimatization period, application of organic amendment is useful to induce formation of healthy seedlings of A. purpurata and Z. spectabile but it may be harmful to the AMF community.

Key words: micropropagation, Glomeromycota, tropical flowers

\section{Introdução}

As plantas ornamentais tropicais possuem características únicas, tais como formas exóticas, durabilidade e alta produtividade, além de beleza peculiar. O mercado tem crescido, pois essas plantas têm boa aceitação e, por outro lado, há saturação na oferta de flores tradicionais (Lamas 2002).
Pernambuco é o maior Estado produtor de flores tropicais no Brasil, com registro de crescimento de cerca de $1.000 \%$ de 1994 a 1999 (Ferreira et al. 2002). Dentre as plantas ornamentais tropicais, duas são comumente produzidas e comercializadas: a Alpinia purpurata (Viell.) Schum (conhecida como alpinia ou Panamá) e Zingiber spectabile Griff. (conhecida como sorvetão).

\footnotetext{
1 Universidade Federal de Pernambuco, Departamento de Micologia, Centro de Ciências Biológicas, CEP 50670-420, Recife, PE, Brasil

2 Embrapa Semi-árido, BR 428, Km 152, Zona Rural, CEP 56302-970, Petrolina, PE, Brasil

3 Autor para correspondência: leonorcmaia@yahoo.com.br
} 
A propagação vegetativa in vitro (micropropagação) permite a obtenção de grande número de indivíduos sadios e geneticamente homogêneos, em curto período de tempo, com alta produtividade e uso de pouco espaço físico (Carvalho 1999). Porém, o último estádio do processo (aclimatação) é crítico, sendo muitas vezes fator limitante, pois as plantas obtidas são transplantadas para substratos desinfestados e transferidas para casa de vegetação (Debergh \& Read 1991), passando assim de uma condição heterotrófica, onde cresciam em condições de umidade e luminosidade controladas, para uma condição autotrófica. É nessa fase que há necessidade da utilização de alternativas para minimizar o estresse produzido pelo transplantio.

Micorrizas, formadas pela maioria das espécies vegetais, são associações entre raízes de plantas com certos fungos do solo. Nessa relação, a planta fornece fotossintatos para o fungo, que em troca cede nutrientes minerais do solo, deixando o vegetal em melhor condição nutricional e mais resistente a estresses de origem biótica e abiótica (Azcón-Aguilar \& Barea 1997). O tipo mais comum de micorriza, sobretudo nas regiões tropicais, é aquele formado pelos fungos micorrízicos arbusculares (FMA).

Os FMA têm sido utilizados na produção de mudas, bem como na aclimatação de plantas micropropagadas de diversas culturas, principalmente fruteiras, com respostas promissoras dos hospedeiros, pois um organismo benéfico está sendo reintroduzido na rizosfera (Lovato et al. 1996). O resultado da associação micorrízica é dependente da espécie de planta, do isolado de FMA, do substrato e das condições ambientais (Sato et al. 1999; Lins et al. 2003).

Os FMA podem influenciar o crescimento de plantas micropropagadas pela redução do bloqueio do crescimento apical (Naqvi \& Mukerji 1998) e pela redução da relação entre parte aérea e radicular (Quatrini et al. 2003), afetando ainda a uniformidade (Rapparini et al. 1994), a sobrevivência (Varma \& Schuepp 1994), e diminuindo o período de aclimatação (Salamanca et al. 1992). Além desses efeitos benéfícos, a micorrização aumenta a absorção de nutrientes, melhorando o estado geral da planta e, conseqüentemente, o crescimento, como observado em mudas de morangueiro, Fragaria ananassa Duch. (Borkowska 2002), bananeira, Musa spp. cv. Grand naine (Declerck et al. 2002) e macieira, Prunus prunifolia (Wild.) Borkh. (Locatelli \& Lovato 2002).

Os substratos utilizados na aclimatação podem influenciar a associação micorrízica e o desenvol- vimento da planta, sendo muitas vezes favoráveis, quando aplicados em doses adequadas, como relatado em macieira, Malus pumila L. (Schubert \& Lubraco 2000), bambu, Dendrocalamus asper Backer ex K. Heyne (Verma \& Arya 1998) e bananeira variedade caipira (Trindade et al. 2003), ou desfavoráveis, como observado em Gentiana verna L. (Sýkorová et al. 2003). Em altas doses de $\mathrm{P}$ (> $250 \mathrm{mg} \mathrm{kg}^{-1}$ ) mudas de citros micorrizadas apresentaram redução de crescimento quando comparadas com o controle (Sena et al. 2004). Assim, é importante a escolha de substratos e respectivos teores que sejam benéficos para determinada cultura, levando-se em consideração que o uso de fontes e doses impróprias podem comprometer os benefícios da micorrização.

O objetivo deste trabalho foi avaliar a influência da inoculação com três espécies de FMA e de substratos compostos na aclimatação de Alpinia purpurata e Zingiber spectabile.

\section{Material e métodos}

Substrato - Foram utilizados três substratos (Tab. 1): (a) solo (camada superficial de latossolo vermelho amarelo, coletado na Estação Experimental de Bebedouro da Embrapa Semi-Árido); (b) solo, areia e vermicomposto (proveniente da compostagem de esterco bovino misturado com solo, utilizando-se minhocas) $(2: 2: 1 \mathrm{v} / \mathrm{v})$; (c) solo e vermicomposto (3:1 $\mathrm{v} / \mathrm{v}$ ), previamente (15 dias) desinfestados com Bromex ${ }^{\circledR}(98 \%$ brometo de metila e $2 \%$ de cloropicrina).

Material vegetal - Plântulas micropropagadas de Alpinia purpurata e Zingiber spectabile, originadas de planta matriz, foram multiplicadas em meio MS (Murashige \& Skoog 1962) suplementado com 4 mg/L de BAP (6-benzilaminopurina) no Laboratório de Biotecnologia da Embrapa Semi-Árido e transplantadas, no início do período de aclimatação, para sacos pretos contendo $2 \mathrm{~kg}$ de cada substrato.

FMA - Foram utilizados propágulos de Gigaspora albida Becker \& Hall (UFPE 01), Glomus etunicatum Becker \& Gerdemann (UFPE 06) e Acaulospora longula Spain \& Schenck (UFPE 21), mantidos no Banco de Inóculo de FMA da Universidade Federal de Pernambuco (UFPE). O inóculo foi multiplicado em vasos com areia e vermiculita $(1: 1 \mathrm{v} / \mathrm{v})$ irrigados com solução nutritiva de Hoagland \& Arnon, modificada por Jarstfer \& Sylvia (1992), e suplementada com Tris- $\mathrm{HCl}(\mathrm{pH} 6,5)$, tendo como hospedeiro Panicum miliaceum L. Após multiplicação, o número 
Tabela 1. Quantidade de nutrientes e matéria orgânica (MO), valor de pH, capacidade de troca catiônica (CTC), extrato saturado (CE) e granulometria dos substratos utilizados na aclimatação de Alpinia purpurata (Viell.) Schum e Zingiber spectabile Griff.

\begin{tabular}{|c|c|c|c|c|c|c|c|c|c|c|c|c|}
\hline Substratos & Areia & $\begin{array}{l}\text { Silte } \\
(\%)\end{array}$ & Argila & $\begin{array}{c}\mathrm{pH} \\
\left(\mathrm{H}_{2} \mathrm{O}-1: 2\right.\end{array}$ & $\begin{array}{c}P \\
\operatorname{lg~dn}\end{array}$ & $\mathrm{Na}$ & $\begin{array}{l}\mathrm{K} \\
(\mathrm{cm}\end{array}$ & $\begin{array}{c}\mathrm{Ca} \\
\left.\mathrm{m}^{-3}\right)\end{array}$ & $\mathrm{Mg}$ & $\begin{array}{c}\mathrm{CE} \\
(\mathrm{dS} / \mathrm{m})\end{array}$ & $\begin{array}{c}\text { CTC } \\
\mathrm{cmol}_{\mathrm{c}} \mathrm{dm}\end{array}$ & $\begin{array}{c}\mathrm{MO} \\
\left(\mathrm{g} \mathrm{kg}^{-1}\right)\end{array}$ \\
\hline $\mathrm{s}$ & 82 & 13 & 5 & 7,1 & 21 & 0,07 & 1,10 & 4,7 & 1,3 & 1,04 & 9,64 & 13,3 \\
\hline s:v (25\%) & 72 & 23 & 5 & 7,1 & 209 & 0,15 & 1,50 & 9,0 & 5,5 & 1,87 & 18,95 & 92,5 \\
\hline s:a:v $(20 \%)$ & 84 & 12 & 4 & 7,1 & 192 & 0,14 & 1,20 & 7,3 & 4,5 & 1,23 & 14,79 & 69,5 \\
\hline
\end{tabular}

$\mathrm{s}=$ solo; $\mathrm{s}: \mathrm{v}=$ solo e vermicomposto; $\mathrm{s}: \mathrm{a}: \mathrm{v}=$ solo, areia e vermicomposto

de propágulos infectivos de cada FMA foi avaliado pelo método de Feldmann \& Idczak (1994). As plântulas foram inoculadas no momento do transplantio para condição ex vitro em potes ( $2 \mathrm{~kg}$ ), sendo o solo-inóculo colocado na região próxima às raízes. Foram aplicados cerca de 1.500 propágulos infectivos de FMA por vaso, no respectivo tratamento. Com isso, as plântulas foram separadas em quatro grupos, sendo um não inoculado e três inoculados separadamente com os fungos mencionados.

Delineamento experimental - Para cada espécie vegetal, o delineamento experimental foi do tipo inteiramente casualizado, em arranjo fatorial de: três substratos (solo, solo e vermicomposto (25\%)-3:1 e solo, areia e vermicomposto (20\%)-2:2:1) $\times$ quatro tratamentos de inoculação (não inoculado e inoculado separadamente com G. albida, G. etunicatum e A. longula) com oito repetições, totalizando 96 parcelas experimentais por espécie vegetal.

Condições experimentais - $\mathrm{O}$ experimento foi realizado na casa de vegetação da Embrapa Semi-Árido, em Petrolina, Pernambuco, sob condições ambientais controladas (temperatura: $27 \pm 2{ }^{\circ} \mathrm{C}$; umidade relativa: $75 \%$; luminosidade: 250 a $\left.560 \mu \mathrm{mol} . \mathrm{m}^{-2} \cdot \mathrm{s}^{-1}\right)$.

Avaliação - Após 70 e 100 dias, respectivamente, em sorvetão e em alpinia, foram avaliados: altura, número de folhas e perfilhos, taxa de sobrevivência, massa seca da parte aérea e subterrânea, área foliar, colonização micorrízica e densidade de esporos de FMA. A biomassa seca foi obtida após secagem em estufa $\left(65^{\circ} \mathrm{C}\right)$ até peso constante. A colonização micorrízica foi estimada pelo método da interseção dos quadrantes (Giovannetti \& Mosse 1980) em raízes coradas com Clorazol Black, 0,03\% (Brundett et al. 1984). Os esporos de FMA foram extraídos do solo por decantação e peneiramento úmido seguido por centrifugação em água e sacarose (Gerdemann \& Nicolson 1963; Jenkins 1964) e quantificados em estereomicroscópio (40x). A área foliar foi estimada utilizando-se o aparelho Li 3100 (LI-Cor Inc. Lincon, Neb., USA).

Análise estatística - Os dados foram submetidos à análise de variância e as médias comparadas pelo teste de Tukey a 5\% de probabilidade, utilizando-se o Programa Statistica (Statsoft 1997). Para análise, valores em percentual e em número de esporos foram transformados em arcoseno $\mathrm{x} / 100$ e de $\sqrt{x}+2,5$, respectivamente.

\section{Resultados e discussão}

Nenhuma das espécies de FMA utilizadas influenciou de forma significativa o crescimento das plantas; diferenças estatísticas foram observadas em relação aos substratos utilizados, com o vermicomposto favorecendo o desenvolvimento das duas espécies vegetais (Tab. 2). Resultados semelhantes foram encontrados por Lovato et al. (1994) em cereja selvagem (Prunus avium L.) inoculada com Glomus intraradices Schenck \& Smith, onde apenas o substrato influenciou o desenvolvimento vegetativo. Por outro lado, em Gentiana verna L. nem a inoculação com FMA nem o uso de adubos orgânicos alterou a produção de biomassa vegetal (Sýkorová et al. 2003).

Os benefícios da micorrização são variados e dependentes do genótipo da planta e do fungo, modulados pelas condições edafoclimáticas (Lovato et al. 1996). Apesar de existirem relatos de benefícios da aplicação de FMA em plantas ornamentais, como Gerbera sp. (Sato et al. 1999), Lilium sp. (Varshney et al. 2002) e Hydrangea sp. (Varma \& Schuepp 1994), tanto A. purpurata quanto $Z$. spectabile não foram beneficiados pela inoculação com FMA. É possível que essa duas espécies não sejam responsivas aos FMA, pelo menos nos estádios iniciais do crescimento. Por outro lado, os elevados teores de P, especialmente nos tratamentos adubados, podem ter contribuído 
Tabela 2. Efeito do substrato sobre variáveis de crescimento em mudas de Alpinia purpurata (Viell.) Schum (alpinia) e Zingiber spectabile Griff. (sorvetão) micropropagadas e cultivadas em solo (s), solo e vermicomposto (s:v/ 3:1) e solo, areia e vermicomposto (s:a:v/ 2:2:1), após 70 (sorvetão) e 100 dias (alpinia) de aclimatação $(\mathrm{n}=8)$

\begin{tabular}{|c|c|c|c|}
\hline & \multicolumn{3}{|c|}{ Substratos } \\
\hline & s & s:v $(25 \%)$ & s:a:v $(20 \%)$ \\
\hline & \multicolumn{3}{|c|}{ Altura (cm) } \\
\hline Alpinia purpurata & $18,14 \mathrm{a}$ & $21,31 \mathrm{a}$ & $19,98 \mathrm{a}$ \\
\hline Zingiber spectabile & $11,91 \mathrm{c}$ & $31,35 \mathrm{a}$ & $24,36 \mathrm{~b}$ \\
\hline $\begin{array}{l}\text { Alpinia purpurata } \\
\text { Zingiber spectabile }\end{array}$ & $\begin{array}{r}12,63 \mathrm{~b} \\
6,28 \mathrm{c}\end{array}$ & $\begin{array}{l}\text { Número de folhas } \\
26,55 \mathrm{a} \\
17,71 \mathrm{a}\end{array}$ & $\begin{array}{l}25,87 \mathrm{a} \\
12,25 \mathrm{~b}\end{array}$ \\
\hline $\begin{array}{l}\text { Alpinia purpurata } \\
\text { Zingiber spectabile }\end{array}$ & $\begin{array}{l}2,50 \mathrm{~b} \\
0,78 \mathrm{c}\end{array}$ & $\begin{array}{l}\text { Número de perfilhos } \\
\qquad \begin{array}{l}5,98 \mathrm{a} \\
2,59 \mathrm{a}\end{array}\end{array}$ & $\begin{array}{l}5,69 \mathrm{a} \\
1,90 \mathrm{~b}\end{array}$ \\
\hline $\begin{array}{l}\text { Alpinia purpurata } \\
\text { Zingiber spectabile }\end{array}$ & $\begin{array}{l}0,69 \mathrm{~b} \\
0,24 \mathrm{c}\end{array}$ & $\begin{array}{c}\text { Massa seca da parte aérea }(\mathrm{g}) \\
1,59 \mathrm{a} \\
1,51 \mathrm{a}\end{array}$ & $\begin{array}{l}1,47 \mathrm{a} \\
0,77 \mathrm{~b}\end{array}$ \\
\hline $\begin{array}{l}\text { Alpinia purpurata } \\
\text { Zingiber spectabile }\end{array}$ & $\begin{array}{l}0,08 \mathrm{~b} \\
0,14 \mathrm{c}\end{array}$ & $\begin{array}{c}\text { Massa seca da parte subterrânea }(\mathrm{g}) \\
0,23 \mathrm{a} \\
0,35 \mathrm{a}\end{array}$ & $\begin{array}{l}0,27 \mathrm{a} \\
0,23 \mathrm{~b}\end{array}$ \\
\hline $\begin{array}{l}\text { Alpinia purpurata } \\
\text { Zingiber spectabile }\end{array}$ & $\begin{array}{r}105,73 \mathrm{~b} \\
59,12 \mathrm{c}\end{array}$ & $\begin{array}{c}\text { Área foliar }\left(\mathrm{cm}^{2}\right) \\
254,09 \mathrm{a} \\
360,72 \mathrm{a}\end{array}$ & $\begin{array}{l}243,61 \mathrm{a} \\
214,08 \mathrm{~b}\end{array}$ \\
\hline
\end{tabular}

Médias seguidas da mesma letra na linha não diferem entre si pelo teste de Tukey (5\%).

para ausência de respostas produzidas por FMA.

Nas mudas de Z. spectabile, o substrato com maior quantidade de vermicomposto $(25 \%)$ resultou em melhores respostas de crescimento em todas as variáveis, diferindo significativamente dos outros dois tratamentos de substrato, que também diferiram entre si, sendo o crescimento favorecido pela presença de vermicomposto. As mudas de A. purpurata cultivadas nos dois substratos adubados com vermicomposto (nas proporções de 20 e $25 \%$ ) também apresentaram maior desenvolvimento, com exceção da altura, do que o observado no tratamento sem adição de vermicomposto (Tab. 2). O solo, sem vermicomposto, foi o substrato menos favorável ao crescimento das espécies estudadas, com os resultados diferindo significativamente, para a maioria das variáveis de crescimento, dos obtidos nos demais substratos testados.

Não houve interações significativas entre a inoculação de FMA e os substratos, tanto para A. purpurata quanto para $Z$. spectabile. Do mesmo modo, em pessegueiros (Prunus persica (L.) Batsch. $\times$ Prunus amygdalus Stokes.) micropropagados, Estaun et al. (1999) observaram que três meses após a inoculação não havia interações significativas entre
FMA e o substrato de cultivo.

As altas doses de vermicomposto (20\% e $25 \%$ ) utilizadas podem ter inibido o estabelecimento da simbiose ativa, uma vez que os FMA inoculados não beneficiaram os hospedeiros testados. Trindade et al. (2003) observaram que o uso de doses reduzidas de esterco (5\%) favoreceu a atuação de Gigaspora margarita Becker \& Hall sobre o crescimento de bananeiras micropropagadas.

Algumas plantas necessitam de mais tempo para estabelecimento de simbiose efetiva, principalmente quando se utiliza substratos ricos em nutrientes, sendo este um fator importante a ser considerado na utilização de FMA. Em mudas de pessegueiro, respostas positivas em relação a substratos e à inoculação micorrízica foram registradas somente após sete meses, nas plantas associadas a $G$. intraradices, que apresentaram aumento de $40 \%$ em relação ao controle; aos 14 meses, todas as plantas inoculadas foram maiores que o controle, independentemente do substrato testado (Estaun et al. 1999). De modo similar, Fortuna et al. (1996) observaram que plantas micorrizadas de macieira (Prunus prunifolia (Wild.) Borkh.) e de ameixeira (Prunus cerasifera Ehrh.) apresentavam 
crescimento similar ao controle não inoculado aos dois meses, com o efeito da micorrização sendo observado apenas depois de seis meses de iniciado o experimento. Estes resultados mostram que o efeito benéfico da simbiose pode aparecer em estádios mais tardios do desenvolvimento da planta, principalmente em substratos ricos em nutrientes. No presente experimento não houve possibilidade de estender a duração do experimento, pois as mudas estavam sendo aclimatadas e no tempo estabelecido para avaliação, plantas de ambas as espécies vegetais atingiram o tamanho necessário para o transplantio ao campo.

A percentagem de sobrevivência das mudas de Z. spectabile foi de $100 \%$ em todos os tratamentos com FMA e substrato, com exceção daquele com G. etunicatum em solo, onde apenas $85 \%$ das plantas sobreviveram. Isto indica que a inoculação com este fungo pode representar dreno adicional de fotossintatos, sem trazer benefícios para o vegetal associado, como observado por Locatelli \& Lovato (2002), em mudas micropropagadas de macieira inoculadas com mistura de Scutellospora pellucida (Nicol. \& Schenck) Walker \& Sanders, G. etunicatum e Glomus sp.

Mudas de A. purpurata tiveram grande variação na taxa de sobrevivência, sendo esta diferenciada entre os substratos e entre os fungos. No tratamento sem FMA, a sobrevivência nos substratos com vermicomposto foi de $100 \%$ e em solo $87,5 \%$. As mudas inoculadas com G. albida tiveram 50, 87 e $100 \%$, com G. etunicatum 25, 75 e $75 \%$ e com $A$. longula 62, 87 e 100\% de sobrevivência nos substratos solo, solo:vermicomposto e solo:areia:vermicomposto, respectivamente. Nem sempre a presença de FMA influencia a sobrevivência de mudas, como observado em ameixeiras micropropagadas (Estaun et al. 1996).

Embora o uso de vermicomposto tenha favorecido o desenvolvimento das plantas, seu efeito sobre a colonização micorrízica foi reduzida em A. purpurata, com formação de estruturas fúngicas em quantidade significativamente superior nos tratamentos apenas com solo, sem adubo em relação aos demais tratamentos (Tab. 3). A colonização micorrízica mais pronunciada $(40,3 \%)$ foi formada apenas por G. albida em A. purpurata, no tratamento não adubado, sendo que a colonização pelos outros fungos significativamente menor $(33,12$ e $28,33 \%$, respectivamente com A. longula e G. etunicatum). Nos tratamentos com adição de vermicomposto, a colonização produzida pelos três FMA não diferiu (Tab. 3). Em contrapartida, as mudas de Z. spectabile associadas a A. longula tiveram a colonização micorrízica visivelmente prejudicada pela adubação orgânica, o que não ocorreu nos demais tratamentos de FMA (Tab. 4). De modo geral, a colonização produzida em $Z$. spectabile foi baixa $(<10 \%$, com exceção de tratamento com A. longula) e provavelmente por isso não se detectou possíveis diferenças na atuação dos FMA.

De modo geral, altos níveis de $\mathrm{P}$ no solo inibem a colonização e o desenvolvimento do FMA, bem como o estabelecimento da simbiose (Schubert \& Martinelli 1988). No presente estudo, os elevados teores de $P$ nos substratos com vermicomposto (Tab. 1) pode ter comprometido o estabelecimento do fungo na raiz. Varshney et al. (2002) observaram que houve redução na colonização micorrízica em plântulas de Lilium sp., quando cultivadas em substrato com elevados teores de $\mathrm{P}\left(14,6 \mathrm{mg} \mathrm{kg}^{-1}\right)$.

Tabela 3. Colonização micorrízica e produção de esporos de FMA associados a Alpinia purpurata (Viell.) Schum cultivada em solo (s), solo e vermicomposto (s:v) e solo, areia e vermicomposto (s:a:v), após 100 dias de aclimatação $(\mathrm{n}=8)$.

\begin{tabular}{|c|c|c|c|}
\hline & \multicolumn{3}{|c|}{ Substratos } \\
\hline & s & s:v $(25 \%)$ & s:a:v $(20 \%)$ \\
\hline & \multicolumn{3}{|c|}{ Colonização micorrízica (\%) } \\
\hline Gigaspora albida & $40,30 \mathrm{aA}$ & $1,37 \mathrm{bA}$ & $0,59 \mathrm{bA}$ \\
\hline Glomus etunicatum & $28,33 \mathrm{aB}$ & $2,12 \mathrm{bA}$ & $2,42 \mathrm{bA}$ \\
\hline \multirow[t]{2}{*}{ Acaulospora longula } & $33,12 \mathrm{aB}$ & $0,53 \mathrm{bA}$ & $0,40 \mathrm{bA}$ \\
\hline & \multicolumn{3}{|c|}{ Número de esporos $\left(50 \mathrm{~g}^{-1}\right.$ solo $)$} \\
\hline Gigaspora albida & $7,25 \mathrm{aB}$ & $0,42 \mathrm{aA}$ & $0,50 \mathrm{aA}$ \\
\hline Glomus etunicatum & $5,50 \mathrm{aB}$ & $0,33 \mathrm{aA}$ & $0,83 \mathrm{aA}$ \\
\hline Acaulospora longula & $117,40 \mathrm{aA}$ & $0,28 \mathrm{bA}$ & $0,12 \mathrm{bA}$ \\
\hline
\end{tabular}

Médias seguidas da mesma letra, minúsculas na linha e maiúsculas na coluna, não diferem entre si pelo teste de Tukey (5\%). 
Tabela 4. Colonização micorrízica e produção de esporos de FMA associados a Zingiber spectabile Griff. cultivada em solo (s), solo e vermicomposto (s:v) e solo, areia e vermicomposto (s:a:v), após 70 dias de aclimatação $(\mathrm{n}=8)$.

\begin{tabular}{|c|c|c|c|}
\hline & \multicolumn{3}{|c|}{ Substratos } \\
\hline & $\mathrm{s}$ & $\mathrm{s}: \mathrm{v}(25 \%)$ & s:a:v (20\%) \\
\hline & \multicolumn{3}{|c|}{ Colonização micorrízica (\%) } \\
\hline Gigaspora albida & $9,39 \mathrm{aA}$ & $2,35 \mathrm{aA}$ & $0,37 \mathrm{aA}$ \\
\hline Glomus etunicatum & $5,74 \mathrm{aA}$ & $1,05 \mathrm{aA}$ & $0,14 \mathrm{aA}$ \\
\hline \multirow[t]{2}{*}{ Acaulospora longula } & $18,51 \mathrm{aA}$ & $0,04 \mathrm{bA}$ & $0,21 \mathrm{bA}$ \\
\hline & \multicolumn{3}{|c|}{ Número de esporos (50 $\mathrm{g}^{-1}$ solo) } \\
\hline Gigaspora albida & $2,12 \mathrm{aB}$ & $0,50 \mathrm{aA}$ & $0,42 \mathrm{aA}$ \\
\hline Glomus etunicatum & $0,12 \mathrm{aB}$ & $0,87 \mathrm{aA}$ & $0,37 \mathrm{aA}$ \\
\hline Acaulospora longula & $25,25 \mathrm{aA}$ & $0,75 \mathrm{bA}$ & $0,87 \mathrm{bA}$ \\
\hline
\end{tabular}

Médias seguidas da mesma letra, minúsculas na linha e maiúsculas na coluna, não diferem entre si pelo teste de Tukey (5\%).

Houve correspondência entre a reduzida colonização e a ineficiência dos FMA utilizados. Em certos casos, mesmo produzindo elevada taxa de colonização das raízes o FMA não beneficiou o hospedeiro (Lovato et al. 1994), o que também ocorreu no presente estudo: as plantas de A. purpurata cultivadas em solo, sem adubo, apresentaram-se bem colonizadas, mas não tiveram o crescimento beneficiado pela presença do fungo micorrízico. Resultados semelhantes foram obtidos por Lovato et al. (1992) em mudas micropropagadas de videira (Vitis vinifera L.) e de abacaxizeiro (Ananas comosus (L.) Merril.) inoculadas com AGC (inoculante comercial), as quais apresentaram elevada taxa de colonização micorrízica (93\%), sem o conseqüente aumento no crescimento.

Nas duas espécies ornamentais estudadas, a presença de vermicomposto reduziu a esporulação de A. longula, enquanto os demais FMA tiveram a reprodução inalterada pela adição do adubo orgânico (Tab. 3 e 4). A adição de doses adequadas de matéria orgânica no substrato pode estimular a esporulação de FMA (Zambolim et al. 1992). A redução na reprodução de A. longula pode indicar sensibilidade deste fungo aos elevados teores de matéria orgânica e $\mathrm{P}$ presentes nos substratos com vermicomposto (Tab. 1). Esse comportamento é reforçado pela elevada esporulação desta espécie em solo sem adubo, em relação aos demais FMA estudados (Tab. 3 e 4). Ressaltando ainda, que representantes de Acaulosporaceae não têm sido referidas em solos submetidos à adubação orgânica (Douds Jr. et al. 1997).

Como observado, a aplicação dos FMA testados não se apresenta como alternativa para reduzir o tempo de aclimatação e aumentar o crescimento de mudas micropropagadas de alpinia e de sorvetão, enquanto o uso de substratos orgânicos favorece a formação de mudas dessas espécies ornamentais. Por outro lado, foi observado efeito negativo do vermicomposto sobre os FMA, sendo necessário portanto avaliar a relação custo $\times$ benefício da adição desse composto, considerando os danos que pode causar à comunidade de FMA, importante componente da microbiota do solo. Assim, a estratégia a ser adotada no campo deverá levar em conta que a adição de propágulos de FMA na área de cultivo e será mais efetiva se no substrato de produção não se incluir vermicomposto em doses elevadas.

\section{Agradecimentos}

À CAPES, pela concessão de Bolsas de Formação de Pesquisador (ME e DO) a Maryluce Albuqueruue da Silva e Fábio Sérgio Barbosa da Silva; ao $\mathrm{CNPq}$, pelo apoio financeiro e Bolsa de Produtividade concedida a Leonor Costa Maia; ao Laboratório de Biotecnologia e Microbiologia da Embrapa Semi-Árido, pelo apoio logístico para o desenvolvimento do trabalho.

\section{Referências bibliográficas}

Azcón-Aguilar, C. \& Barea, J.M. 1997. Applying mycorrhiza biotechnology to horticulture: significance and potentials. Scientia Horticulturae 68: 1-24.

Borkowska, B. 2002. Growth and photosynthetic activity of micropropagated strawberry plants inoculated with endomycorrhizal fungi (AMF) and growing under drought stress. Acta Physiologiae Plantarum 24(4): 365-370. 
Brundett, M.C.; Piché, Y. \& Peterson, R.L. 1984. A new method for observing the morphology of vesiculararbuscular mycorrhizae. Canadian Journal of Botany 62: 2128-2134.

Carvalho, J.M.F.C. 1999. Técnicas de micropropagação. Documento 64. Embrapa Algodão, Campina Grande, Paraíba.

Debergh, P.C. \& Read, P.E. 1991. Micropropagation. Pp. 1-13. In: P.C. Debergh \& R.H. Zimmerman (eds.). Micropropagation: Technology and application. London, Kluwer Academic Publishers, Dordrecht, Netherlands.

Douds Jr., D.D.; Galvez, L.; Franke-Snyder, M.; Reiders, C. \& Drinkwater, L.E. 1997. Effect of compost addition and crop rotation point upon VAM fungi. Agriculture, Ecosystems and Environment 65: 257-266.

Declerck, S.; Risede, J.M. \& Delvaux, B. 2002. Greenhouse response of micropropagated bananas inoculated with "in vitro" monoxenically produced arbuscular mycorrhizal fungi. Scientia Horticulturae 93: 301-309.

Estaun, V.; Calvet, C. \& Camprubi, A. 1996. Arbuscular mycorrhizae and growth enhancement of micropropagated Prunus rootstock in different soilless potting mixes. Agricultural Science in Finland 3: 263-267.

Estaun, V.; Calvet, C.; Camprubi, A. \& Pinochet, J. 1999. Long-term effects of nursery starter substrate and AM inoculation of micropropagated peach $\mathrm{x}$ almond hybrid rootstock GF677. Agronomie 19: 483- 489.

Feldmann, F. \& Idczak, E. 1994. Inoculum production of vesicular-arbuscular mycorrhizal fungi for use in tropical nurseries. Pp.799-817. In: J.R. Norris; D. Read \& A.K. Varma (eds.). Techniques for Mycorrhizal Research. Methods in Microbiology. London, Academic Press.

Ferreira, A.; Souza Filho, I.C. \& Albuquerque, S.R.C. 2002. Panorama global da floricultura em Pernambuco. Pp. 23-59. In: E. Ramos (ed.). Floricultura em Pernambuco. Recife, Edição Sebrae, Série Agronegócio.

Fortuna, P.; Citernesi, S.; Morini, S.; Vitagliano, C. \& Giovannetti, M. 1996. Influence of arbuscular mycorrhizae and phosphate fertilization on shoot apical growth of micropropagated apple and plum rootstocks. Tree Physiology 16: 757-763.

Gerdemann, J.W. \& Nicolson, T.H. 1963. Spores of mycorrhizal Endogone species extracted from soil by wet sieving and decanting. Transactions of the British Mycological Society 46(2): 235-244.

Giovanetti, M. \& Mosse, B. 1980. An evaluation of techniques for measuring vesicular arbuscular mycorrhizal infection in roots. New Phytologist 84: 489-500.

Jarstfer, A.G. \& Sylvia, D.M. 1992. Inoculum production and inoculation strategies for vesicular arbuscular mycorrhizal fungi. Pp. 349-377. In: Soil Microbial Tecnologies: Applications in Agriculture, Forestry and Environmental Management. New York, Ed. B. Metting. Marcel Dekker.

Jenkins, W.R. 1964. A rapid centrifugal-flotation technique for separating nematodes from soil. Plant Disease Report 48(9): 692.
Lamas, A.M. 2002. Floricultura Tropical (técnicas de cultivo). Recife, Edição Sebrae, Série empreendedor.

Lins, G.M.L.; Trindade, A.V. \& Rocha, H.S. 2003. Utilização de Gigaspora margarita em plantas micropropagadas de bananeira em diferentes estádios de enraizamento. Revista Brasileira de Fruticultura 25(1): 143- 147.

Locatelli, L.M. \& Lovato, P.E. 2002. Inoculação micorrízica e aclimatação de dois porta-enxertos de macieira micropropagados. Pesquisa Agropecuária Brasileira 37(2): 177-184.

Lovato, P.E.; Gianinazzi-Pearson, V.; Trouvelot, A. \& Gianinazzi, S. 1996. The state of art of mycorrhizas and micropropagation. Advances in Horticultural Science 10: $46-52$.

Lovato, P.E.; Guillemin, J.P. \& Gianinazzi, S. 1992. Application of commercial arbuscular endomycorrhizal fungal inoculants to the establishment of micropropagated grapevine rootstock and pineapple plants. Agronomie 12: 873-880.

Lovato, P.E.; Hammatt, N.; Gianinazzi-Pearson, V. \& Gianinazzi, S. 1994. Mycorrhization of micropropagated mature wild cherry (Punus avium L.) and common ash (Fraxinus excelsior L.). Agricultural Science in Finland 3: 297-301.

Murashige, T. \& Skoog, F. 1962. A revised medium for rapid growth and bioassays with tobacco tissue cultures. Physiologia Plantarum 15: 473-497.

Naqvi, N.S. \& Mukerji, K.G. 1998. Mycorrhization of micropropagated Leucaena leucocephala (Lam.) de Wit. Symbiosis 24: 103-114.

Quatrini, P.; Gentile, M.; Carimi, F.; Pasquale, F. \& Puglia, A.M. 2003. Effect of native arbuscular mycorrhizal fungi and Glomus mosseae on acclimatization and development of micropropagated Citrus limon (L.) Burm. Journal of Horticultural Science \& Biotechnology 78(1): 39-45.

Rapparini, F.; Baraldi, R.; Bertazza, G.; Branzanti, B. \& Predieri, S. 1994. Vesicular arbuscular mycorrhizal inoculation of micropropagated fruit trees. Journal of Horticultural Science 69(6): 1101-1109.

Salamanca, C.P.; Herrera, M.A. \& Barea, J.M. 1992. Mycorrhizal inoculation of micropropagated woody legumes used in revegetation programmes for desertified Mediterranean ecosystems. Agronomie 12: 869-872.

Sato, A.Y.; Nannetti, D.C.; Pinto, J.E.B.P.; Siqueira, J.O. \& Blank, M.F.A. 1999. Fungos micorrízicos arbusculares no desenvolvimento de mudas de helicônia e gérbera micropropagadas. Horticultura Brasileira 17(1): 25-28.

Schubert, A. \& Lubraco, G. 2000. Mycorrhizal inoculation enhances growth and nutrient uptake of micropropagated apple rootstock during weaning in commercial substrates of high nutrient availability. Applied Soil Ecology 15: 113-118.

Schubert, A. \& Martinelli, A. 1988. Effect of vesicular arbuscular mycorrhizae on growth of "in vitro" propagated Pistacia integerrima. Acta Horticulturae 227: 441-443. 
Sena, J.O.A.; Labate, C.A. \& Cardoso, E.J.B.N. 2004. Caracterização fisiológica da redução de crescimento de mudas de citros micorrizadas em altas doses de fósforo. Revista Brasileira de Ciências do Solo 28: 827-832.

Statsoft, 1997. Statistica for Windows. Tulsa: CD-ROM.

Sýkorová, Z.; Rydlova, J. \& Vosatka, M. 2003. Establishment of mycorrhizal symbiosis in Gentiana verna. Folia Geobotanica 38: 177-189.

Trindade, A.V.; Lins, G.M.L. \& Maia, I.C.S. 2003. Substratos e fungo micorrízico arbuscular em mudas micropropagadas de bananeira na fase de aclimatação. Revista Brasileira de Fruticultura 25(1): 137-142.

Varma, A. \& Schuepp, H. 1994. Positive influence of arbuscular mycorrhizal fungus on in vitro raised Hortensia plantlets. Angewandti Botanik 68: 108-115.
Varshney, A.; Sharma, M.P.; Adholeya, A.; Dhawan, V. \& Srivastava, P.S. 2002. Enhanced growth of micropropagated bulblets of Lilium sp. inoculated with arbuscular mycorrhizal fungi at diferent P fertility levels in an alfisol. Journal of Horticultural Science \& Biotechnology 77(3): 258-263.

Verma, R.K. \& Arya, I.D. 1998. Effect of arbuscular mycorrhizal fungal isolates and organic manure on growth and mycorrhization of micropropagated Dendrocalamus asper plantlets and on spore production in their rhizosphere. Mycorrhiza 8: 113-116.

Zambolim, L.; Reis, M.A. \& Costa, L.M. 1992. Substratos para multiplicação de inóculo do fungo micorrízico vesículo-arbuscular Glomus etunicatum. Fitopatologia Brasileira 17: 28-31. 